\title{
In Vitro Evaluation of a Biomedical-Grade Bilayer Chitosan Porous Skin Regenerating Template as a Potential Dermal Scaffold in Skin Tissue Engineering
}

\author{
Chin Keong Lim, ${ }^{1}$ Ahmad Sukari Halim, ${ }^{1}$ Ismail Zainol, ${ }^{2}$ and Kartini Noorsal ${ }^{3}$ \\ ${ }^{1}$ Reconstructive Sciences Unit, School of Medical Sciences, Universiti Sains Malaysia, Kelantan, 16150 Kubang Kerian, Malaysia \\ ${ }^{2}$ Department of Chemistry, Faculty of Science and Mathematics, Universiti Pendidikan Sultan Idris, \\ 35900 Tanjung Malim, Perak, Malaysia \\ ${ }^{3}$ Advanced Materials Research Centre (AMREC) SIRIM Berhad, Lot 34, Jalan Hi-Tech 2/3, Kulim Hi-Tech Park, \\ Kedah, 09000 Kulim, Malaysia \\ Correspondence should be addressed to Ahmad Sukari Halim, ashalim@kb.usm.my
}

Received 18 May 2011; Revised 10 July 2011; Accepted 10 July 2011

Academic Editor: Shanfeng Wang

Copyright (๑) 2011 Chin Keong Lim et al. This is an open access article distributed under the Creative Commons Attribution License, which permits unrestricted use, distribution, and reproduction in any medium, provided the original work is properly cited.

\begin{abstract}
Chitosan is a copolymer of $N$-acetylglucosamine and glucosamine. A bilayer chitosan porous skin regenerating template (CPSRT) has been developed for skin tissue engineering. The pore size of the CPSRT was assessed using a scanning electron microscopy (SEM). The in vitro cytocompatibility of the CPSRT was tested on primary human epidermal keratinocyte (pHEK) cultures by measuring lactate dehydrogenase (LDH) levels and skin irritation by western blot analysis of the interleukin-8 (IL-8) and tumor necrosis factor- $\alpha$ (TNF- $\alpha$ ) secretions. The ability of the CPSRT to support cell ingrowth was evaluated by seeding primary human dermal fibroblasts (pHDFs) on the scaffold, staining the cells with live/dead stain, and imaging the construct by confocal microscopy (CLSM). The CPSRT with pore sizes ranging from 50 to $150 \mu \mathrm{m}$ was cytocompatible because it did not provoke the additional production of IL- 8 and TNF- $\alpha$ by pHEK cultures. Cultured pHDFs were able to penetrate the CPSRT and had increased in number on day 14. In conclusion, the CPSRT serves as an ideal template for skin tissue engineering.
\end{abstract}

\section{Introduction}

Biopolymers are naturally synthesized compounds that are produced by living organisms. These materials participate in the natural biological cycle and are eventually degraded and reabsorbed by their environment. Chitin, a biopolymer, can be obtained at relatively low cost from the shells of shellfish (mainly crabs, shrimps, and lobsters), which are a waste product of the seafood processing industry [1]. Chitin and derivatives such as chitosan have a natural basicity that provides properties such as biocompatibility, biodegradability, antibacterial activity, heavy metal ion chelation ability, gel-forming properties, hydrophilicity, and remarkable protein affinity [2].

Chitosan is the primary biopolymer derivative of $\mathrm{N}$-deacetylated chitin and consists of $N$-acetylglucosamine and glucosamine. Chitosan possesses an abundance of amino and hydroxyl groups that allow it to be chemically modified by processes such as acylation, $N$-phthaloylation, alkylation, Schiff base formation, reductive alkylation, tosylation, $\mathrm{O}$-carboxymethylation, $\mathrm{N}$-carboxyalkylation, and graft copolymerization $[3,4]$. These modifications allow chitosan's properties to be tailored to a specific application.

Tissue regeneration is a complex biological process that involves inward migration and proliferation of cells into a defect area or scaffold, as well as the secretion of an extracellular matrix to support new tissue formation. Biocompatible tissue engineering constructs can provide cues for cell migration and differentiation to promote wound-healing, tissue formation, and vascular network regrowth. Tissue engineering uses polymer scaffolds to promote cell adhesion, proliferation, and differentiation in vitro. Although chitosan has been used in various types of biocompatible wound dressings, such as films, pastes, sheets, and porous templates 
[5-8], it also has intrinsic wound-healing abilities [9]. A novel biomedical-grade bilayer chitosan porous skin regenerating template (CPSRT) was developed for use as a dermal scaffold for skin tissue engineering to take advantage of these wound-healing abilities as well as the biocompatible nature of chitosan. The pore size of the CPSRT was assessed using SEM analysis. However, the cytocompatibility and skin irritation of the CPSRT were tested in vitro using $\mathrm{pHEK}$ cultures. The in vitro cellular ingrowth into the CPSRT scaffold was evaluated using pHDF and was observed by CLSM.

\section{Materials and Methods}

2.1. Preparation and Microstructure Examination of CPSRT. CPSRT was developed at the Advanced Materials Research Centre (AMREC-SIRIM), Malaysia and in accordance with the methods described by Zainol et al. [10]. Pharmaceuticalgrade chitosan powder with molecular weight at $634 \mathrm{kDa}$ and deacetylation degree (DD) at $89 \%$ was purchased from Hunza Nutriceuticals Sdn Bhd, Perak, Malaysia. Chitosan was dissolved in $1 \%(\mathrm{v} / \mathrm{v})$ acetic acid to prepare a $2 \%(\mathrm{w} / \mathrm{v})$ chitosan solution. About 20\% (w/w) glycerol were added as a plasticizer, followed by neutralization with sodium bicarbonate to achieve a $\mathrm{pH}$ of 6.2. The chitosan solution was then poured into a polytetrafluoroethylene (PTFE) mold and was casted and left dry at room temperature for the preparation of chitosan film. To create the porous structure of the CPSRT, the chitosan solution was frozen at $-20^{\circ} \mathrm{C}$ and lyophilized for 24 hours. The chitosan bilayer CPSRT was fabricated through the attachment of both chitosan film and chitosan sponge using chitosan solution as the glue. The CPSRT was sterilized using ethylene oxide (EO) according to the International Standards Organization (ISO) guidelines (Part 10993-7:1995: Ethylene Oxide Sterilization Residuals).

The microstructure of the CPSRT was then observed via an SEM system. Briefly, the CPSRT was fixed in $2.5 \%$ glutaraldehyde for 1 hour followed by fixation in $2 \%$ Osmiumtetroxid for 30 minutes at room temperature. The CPSRT was then washed two times in distilled water, and the dehydration was performed using a graded series of ethanol (25\%, 50\%, 75\%, and $100 \%$ ethanol for 5 minutes each).

2.2. $p H E K$ and $p H D F$ Cultures. Skin samples were obtained from consenting patients who had undergone elective surgery at Hospital Universiti Sains Malaysia, Kubang Kerian, Kelantan, after being approved by the Human Ethic Committee of Universiti Sains Malaysia.

The epidermal layer was lifted from the dermal layer after incubation in a dispase solution $(2.4$ units $/ \mathrm{mL})$. Keratinocytes were released from the epidermal layer using $0.25 \%$ trypsin-EDTA for 15 minutes at $37^{\circ} \mathrm{C}$. The trypsin was deactivated by adding Dulbecco's minimal Eagle's medium (DMEM) supplemented with 10\% fetal bovine serum (FBS). The pHEKs were centrifuged for 7 minutes at $200 \times g$ and were resuspended in CnT-07 (CELLnTEC Advanced cell system) growth medium. pHEK cells were seeded at a density of $2 \times 10^{5}$ viable cells/mL in culture flasks and were incubated at $37^{\circ} \mathrm{C}$ with $5 \% \mathrm{CO}_{2}$.
The remaining dermal layer was minced into smaller pieces and digested using collagenase type-I $(200 \mathrm{cu} / \mathrm{mL}$ of DMEM) for 12 hours at $37^{\circ} \mathrm{C}$ to extract the pHDFs. The dissociated fibroblasts were washed with DPBS, and the cell pellets were sieved through a $70 \mu \mathrm{m}$ strainer before being centrifuged at $200 \times g$ for 10 minutes. The $\mathrm{pHDF}$ were seeded at $2 \times 10^{5}$ viable cells/mL in DMEM supplemented with $10 \%$ FBS and $1 \%$ penicillin-streptomycin.

2.3. Cytotoxicity of CPSRT by LDH Assay. The pHEK cultures at passage 3 were seeded at a density of $5 \times 10^{4}$ cells $/ \mathrm{mL}$ in 24 well plate. CPSRT was sized into $5 \times 5 \mathrm{~mm}^{2}$ and was washed twice in the CnT-07 medium. The CPSRTs were then placed on $70 \%$ confluent cultures of pHEK in the same manner as a direct-contact test. The experiment was performed for 72 hours on 5 skin samples.

At each time point, $100 \mu \mathrm{L}$ of supernatant was removed and added to an optically clear 96-well flat bottom microplate. This was mixed with $100 \mu \mathrm{L}$ of $\mathrm{LDH}$ reaction mixtures (diaphorase/NAD ${ }^{+}+$iodotetrazolium chloride and sodium lactate) and incubated for 30 minutes at room temperature, as described in the instruction manual of the LDH Cytotoxicity Detection kit (Roche). The optical density (OD) of the test samples and the controls was measured at $490 \mathrm{~nm}$ using an enzyme-linked immunosorbent assay (ELISA) plate reader. The reference wavelength was set to $620 \mathrm{~nm}$. pHEK cultures treated with 1\% triton-X solution served as the positive control, whereas pHEKs with only growth medium served as the negative control. Background absorbance was measured using growth medium alone. The cytotoxicity was expressed as a percentage of the positive control, as shown in the following equation:

Cytotoxicity (\%)

$$
=\frac{(\text { Experimental OD }- \text { Negative control })}{(\text { Positive control }- \text { Negative control })} \times 100 \% \text {. }
$$

2.4. Western Blot Analysis of IL-8 and TNF- $\alpha$ Skin Proinflammatory Cytokines. This experiment was performed according to the western blotting protocols described by Millipore and Coufal et al. [11] with minor modifications. Cultured pHEKs in direct contact with CPSRTs were removed from the 6-well plates at 24 and 72 hours using Accutase.

Protein was extracted from $5 \times 10^{6}$ pelleted cells $(n=3)$ in $1 \mathrm{~mL}$ of RIPA buffer with salt $(0.4 \mathrm{M}$ sodium chloride, $50 \mathrm{mM}$ Tris- $\mathrm{HCl}$ at $\mathrm{pH} 7.4,1 \% \mathrm{NP}-40,0.1 \%$ sodium dodecyl sulphate (SDS), 1\% Na-deoxycholate, $1 \mathrm{mM}$ EDTA and $1 \mathrm{mM}$ phenylmethanesulphonyl fluoride) at $4^{\circ} \mathrm{C}$ on a shaker for 5 hours. The lysed cells were then centrifuged at $15,700 \times g$ for 20 minutes at $4^{\circ} \mathrm{C}$.

A $10 \%$ resolving gel (monomer solution acrylamide, $4 \mathrm{X}$ running gel buffer $\mathrm{pH} 8.8,10 \%$ ammonium persulphate (APS), 10\% SDS, TEMED and $\mathrm{ddH}_{2} \mathrm{O}$ ) was precast for 15 minutes before adding a layer of $7.5 \%$ stacking gel on top (monomer solution acrylamide, $4 \mathrm{X}$ stacking gel buffer at $\mathrm{pH}$ 6.8, $10 \%$ APS, $10 \%$ SDS, TEMED, and $\left.\mathrm{ddH}_{2} \mathrm{O}\right)$. Ten microliters of sample (at $1 \mu \mathrm{g} / \mu \mathrm{L}$ total protein concentration) was 


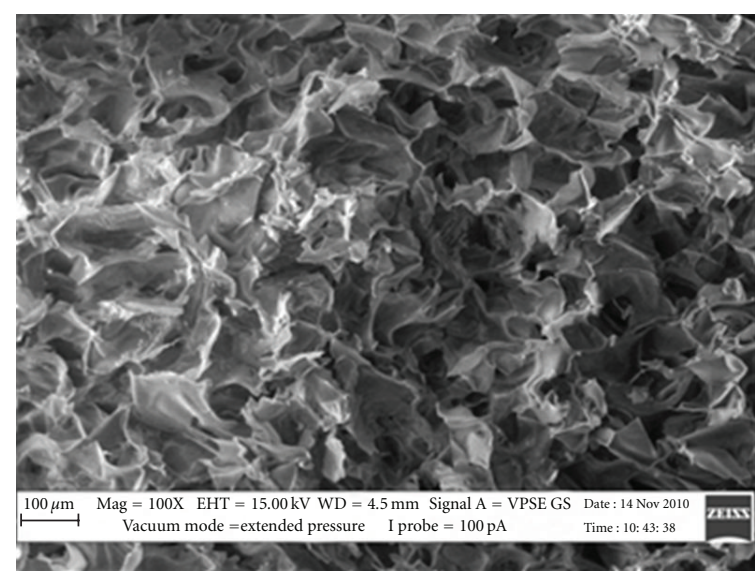

(a)

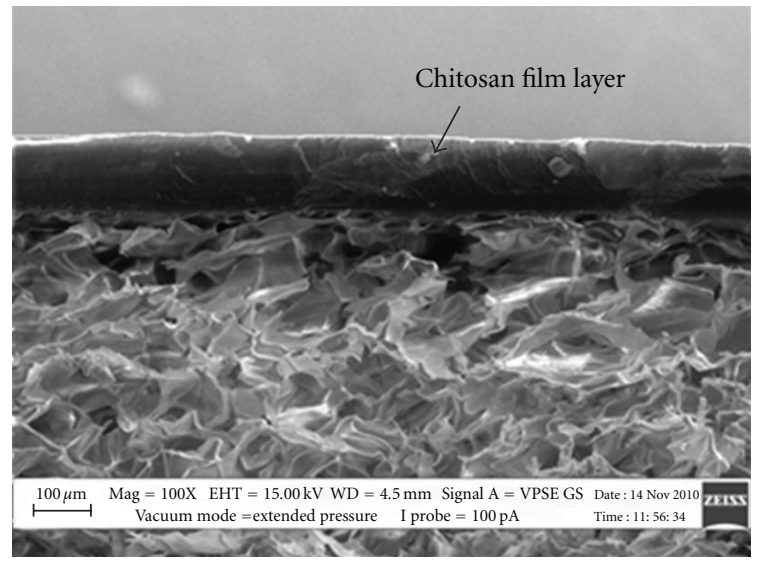

(b)

FIGURE 1: SEM images of CPSRT. (a) Surface of sponge layer. (b) Cross-sectional view of CPSRT that indicates a chitosan film combining a chitosan porous layer.

mixed with $10 \mu \mathrm{L}$ of $2 \mathrm{X}$ protein sample loading buffer. Protein samples were heated for 5 minutes at $96^{\circ} \mathrm{C}$ and loaded into wells, followed by the addition of $5 \mu \mathrm{L}$ of prestained protein ladder in a different well. SDS polyacrylamide gel electrophoresis was performed at $150 \mathrm{~V}$ for 45 minutes. Transblotting was conducted at a constant $13 \mathrm{~V}$ for 90 minutes.

The transblotted polyvinylidene fluoride membrane was blocked with blocking buffer $(5 \%$ w/v nonfat milk, $1 \mathrm{X}$ Tris buffered saline (TBS) and 0.05\% Tween-20) for 1 hour, followed by washing buffer (1X TBS and $0.05 \%$ Tween-20) for 10 minutes. The membranes were then incubated with the following primary antibodies: mouse monoclonal antihuman IL-8 (dilution $1: 1000$ ) (Abcam), TNF- $\alpha$ (dilution $1: 1000)$ (Abcam), and loading control alpha-tubulin (dilution $1: 5000)$ (Abcam) for 120 minutes each. Goat antimouse HRP-conjugated secondary antibody (dilution at $1: 2000$ ) (Sigma-Aldrich) was added and incubated for 1 hour at room temperature. The membrane was then incubated with chemiluminescent horseradish peroxidase substrate for 3 minutes at room temperature before visualization and quantification of band intensity using a Chemilmager 4040 image analyzer. Protein band intensity was scored in arbitrary intensity units (AIUs) and was normalized to the alpha-tubulin loading control. Each experiment was repeated in three skin samples. Cultured pHEK in direct contact with low-density polyethylene (LDPE) served as negative control whereas the organotin-polyvinylchloride (PVC) as positive control.

2.5. Growth of $\mathrm{PHDF}$ within CPSRT Observed via CLSM Microscopy. pHDF cultures at passage 3 were dislodged from culture flasks and seeded at $2 \times 10^{6}$ viable cells $/ \mathrm{mL}$ onto CPSRTs in 96-well plates. After 24 hours, the CPSRTs were transferred to 12-well plates. Growth medium was changed every day to mitigate nutrient depletion. Cultures were evaluated at days 5 and 14 .

Briefly, CPSRTs were transferred back to 96-well plates and washed twice with Dulbecco's phosphate buffered saline (DPBS) before live/dead cell staining. One hundred microliters of live/dead cell reagents (Molecular Probe, Invitrogen) containing calcein $(2 \mu \mathrm{M})$ and ethidium homodimer$1(4 \mu \mathrm{M})$ was added to each CPSRT and incubated for 45 minutes at room temperature. CPSRTs were imaged both in cross-section and on the construct surface under a CLSM with excitation/emission wavelengths at $495 \mathrm{~nm} / 515 \mathrm{~nm}$ for calcein and $495 \mathrm{~nm} / 635 \mathrm{~nm}$ for ethidium-homodimer-1. CPSRTs incubated in growth medium without cultured cells served as the negative control.

2.6. Statistical Analyses. The results are expressed as mean \pm standard deviation (SD). The Wilcoxon signed-rank test was used to assess significance among the different experimental conditions. Differences were regarded as significant at $P \leq 0.05$.

\section{Results}

3.1. Structure of CPSRT. The microstructure of the sponge layer and the cross-sectional view bilayer CPSRT was observed under an SEM (Figure 1). The pore sizes of CPSRT are ranging from 50 to $150 \mu \mathrm{m}$ (Figure 1(a)). The chitosan film layer was intact to the chitosan porous layer, making up a bilayer structure (Figure 1(b)).

3.2. Cytotoxicity of CPSRT In Vitro. Cytotoxicity in vitro was assessed by measuring the amount of LDH released into the supernatant from damaged pHEKs after 72 hours of incubation with CPSRTs, as a percentage of the LDH values in positive controls. The $\mathrm{LDH}$ assay indicated that the CPSRTs displayed no measurable cytotoxic behavior when compared with positive controls at all time points (Figure 2). However, cytotoxicity was noticeably elevated after 48 hours of treatment with CPSRTs compared to the 6-hour CPSRT treatment. The CPSRT cytotoxicity was significantly reduced at 72 hours $(P<0.05)$.

3.3. Proinflammatory Cytokine Expression of CPSRT. IL-8 and TNF- $\alpha$ proteins were expressed by pHEKs at all time 


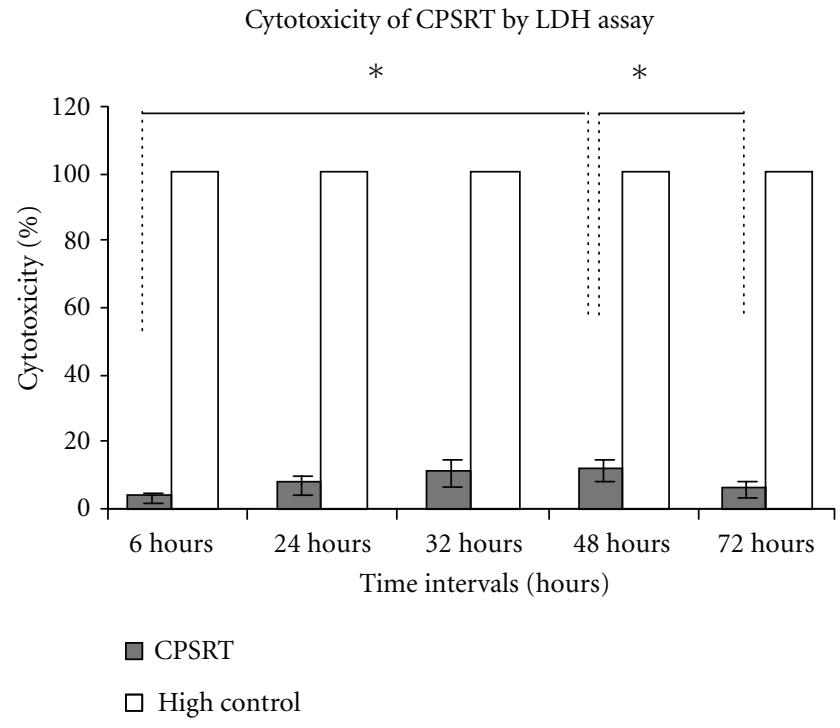

FIgURe 2: Cytotoxicity of CPSRT as determined by the release of LDH from the damaged cells. $n=5$, * for $P \leq 0.05$.

points (Figures 3 and 4). pHEKs treated with organotin-PVC produced the most IL- 8 and TNF- $\alpha$ at 24 hours, and both proteins were noticeably increased at 72 hours $(P<0.05)$. No significant difference in IL-8 expression was observed between pHEKs treated with CPSRT and LDPE at both time points. However, TNF- $\alpha$ expression remained unchanged in the treatment with CPSRTs at both time points and had the lowest value out of all three groups (CPSRT, organotinPVC, LDPE). Although pHEKs treated with LDPE expressed higher TNF- $\alpha$ compared with CPSRT at 24 hours, LDPEinduced TNF- $\alpha$ levels declined at 72 hours.

3.4. Ingrowths of pHDF on CPSRT. The ingrowth of $\mathrm{pHDF}$ cells was observable at day 5 by CLSM (Figure 5). The viable cultured cells propagated in small groups within the CPSRT pores, as shown by the green fluorescence (Figure 5(a)). No significant quantity of dead or apoptosis cells was seen, indicated by the lack of red fluorescence emission. At fourteen days postseeding, the groups of $\mathrm{pHDF}$ cells had proliferated enough to fuse into a larger cell mass that covered the top surface of CPSRT (Figure 5(b)). Furthermore, pHDF cells had also infiltrated and proliferated into the porous structure of CPSRT, as visualized in the crosssectional view (Figure 5(c)).

\section{Discussion}

In this study, a skin-like bilayer porous chitosan scaffold mimicking the thickness of natural skin was created for skin tissue engineering. For skin tissue engineering, pore size for a scaffold is recommended to be within the range between 100 to $200 \mu \mathrm{m}$ [12]. Nevertheless, significant cell proliferation and differentiation were reported for the scaffolds with small pore sizes. Chitosan-gelatin-hydroxyapatite scaffold with pore sizes at the top $(65-80 \mu \mathrm{m})$ and bottom $(10-20 \mu \mathrm{m})$, resulted in proliferation of fibroblasts and keratinocytes [13].
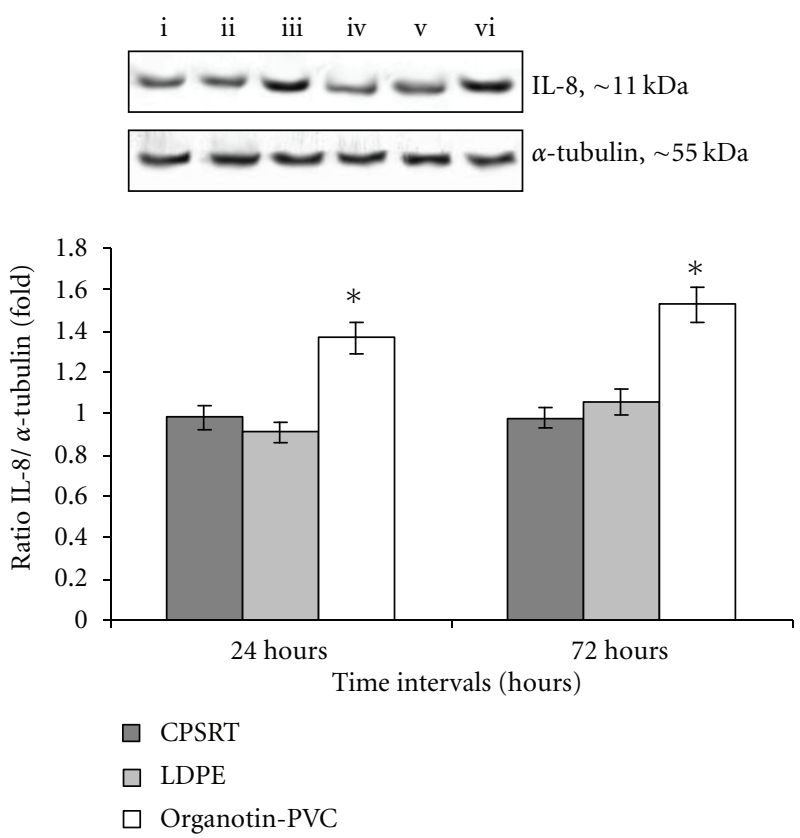

FIGURE 3: Western blot analysis of IL-8 in pHEK cultures. pHEK cultures were treated for 24 hours ((i) CPSRT, (ii) LDPE, and (iii) organotin-PVC) and 72 hours ((iv) CPSRT, (v) LDPE, and (vi) organotin-PVC) $(n=3)$. *For $P \leq 0.05$ compared with both CPSRT and LDPE.
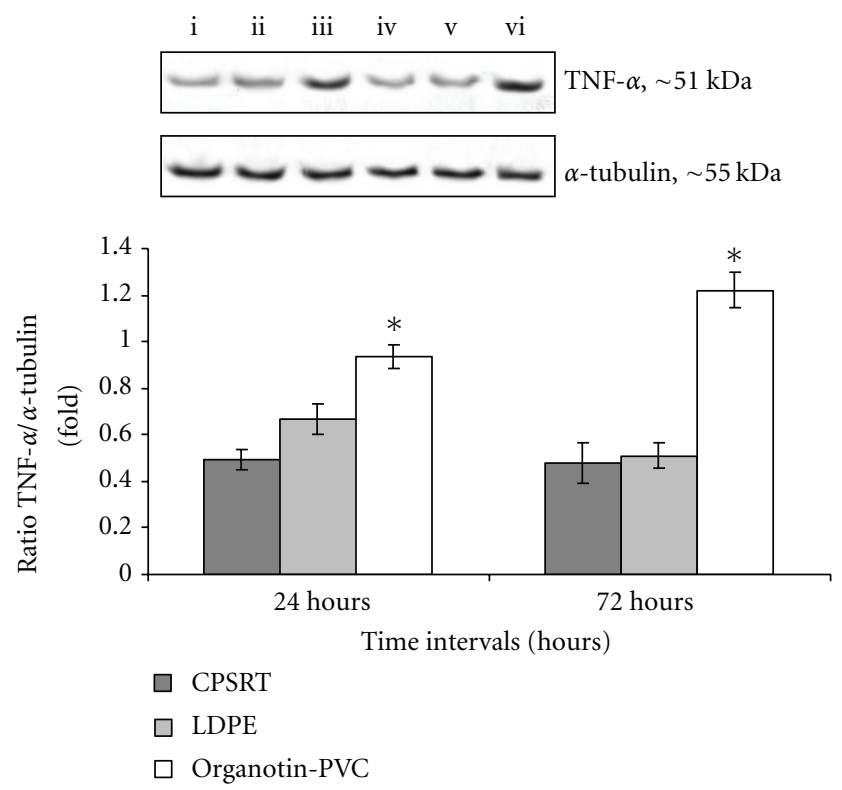

FIGURE 4: TNF- $\alpha$ protein expression in western blot analysis. pHEK cultures were treated for 24 hours [(i) CPSRT, (ii) LDPE and (iii) organotin-PVC] and 72 hours [(iv) CPSRT, (v) LDPE and (vi) organotin-PVC] $(n=3)$. * For $P \leq 0.05$ compared with CPSRT.

Similarly, lyophilized chitosan scaffold coated with collagen (40-100 $\mu \mathrm{m}$ pore sizes) was shown to have interconnected structure, which enhanced cell proliferation and woundhealing without inflammation [14].

However, although chitosan is biocompatible, to ensure the CPSRT fabrication process did not alter its natural 


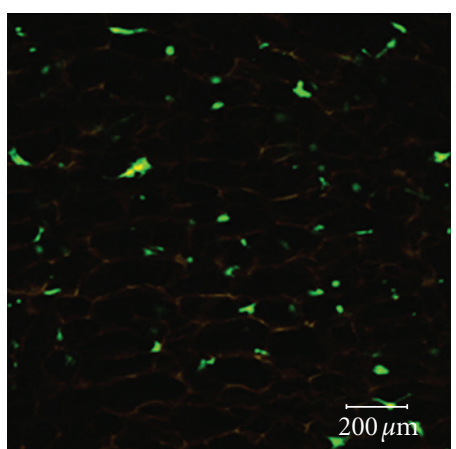

(a)

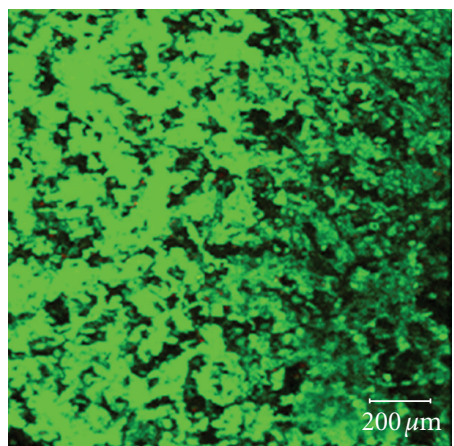

(b)

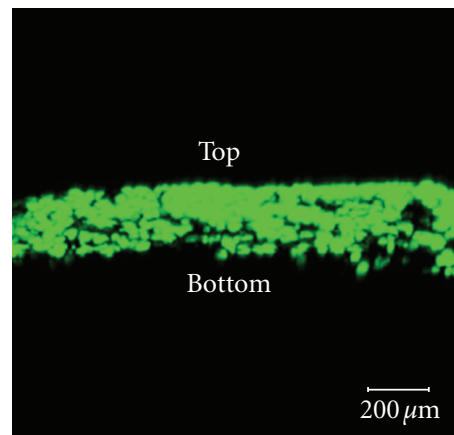

(c)

FIGURE 5: Live/dead cell staining of pHDF cultures seeded on CPSRTs. (a) Three-dimensional (3D) surfaces were scanned using a CLSM at day 5. (b) Viable pHDFs (green) were confluent at day 14. (c) Cross-sectional view of a CPSRT seeded with pHDFs at day 14.

biocompatibility, in vitro models of cytotoxicity were used to evaluate the construct. In vitro cytotoxicity models are often used to screen for potential harmful effects of chemical compounds because they have good reproducibility and sensitivity, while also minimizing the use of animals. Moreover, cellular cytotoxicity systems are better predictive tools for human toxicity than are whole organism models $[5,6,15,16]$.

By using primary human cell cultures, in this case pHEKs, these results should also better reflect the cytotoxicity outcomes expected in humans, compared with results from transformed cell lines, fibroblasts, or animal cell lines $[6,7,17,18]$. $\mathrm{LDH}$ is a stable cytoplasmic enzyme present in most cells that is released when the plasma membrane is damaged or ruptured. Therefore, measuring LDH levels is a highly sensitive and accurate measure of cytotoxicity [19]. The CPSRTs in this study did not cause significant cytotoxicity compared with the positive control. However, the reduction in LDH observed at 72 hours may be attributed to chitosan-induced proliferation and cell growth, which is corroborated by the findings of Cho et al. [20]. Chitosan's oligomers $N$-acetylglucosamine and glucosamine have been implicated in enhancing cell growth. Glucosamine has been reported to be important for detoxification in the liver and kidneys, as well as in anti-inflammatory, hepatoprotective, antireactive, and antihypoxic activities [21, 22]. $N$-acetylglucosamine is a major component of dermal tissue that is essential for repairing scar tissue and is present in large quantity in the early phase of woundhealing. It has been effective in modulating keratinocyte cellular adhesion, proliferation, and differentiation, leading to the normalization of stratum corneum exfoliation [23]. Therefore, it is possible that CPSRTs could promote the proliferation of keratinocytes by functioning as a controlled delivery source for $N$-acetylglucosamine and glucosamine to the in vitro wound-like model in this study.

Because the CPSRT is intended to be a scaffold for skin transplantation, it should avoid irritating epidermal transplantation sites to avoid failure of the bioengineered skin graft. Keratinocytes are the primary cell type in the epidermis and serve as a major contributor of epidermal cytokines, particularly pro-inflammatory cytokines, that act as the first line of defense to protect the body from invaders [24]. Many of the currently identified cytokines, such as IL6 , IL-7, IL- 8 , and TNF- $\alpha$, are produced by keratinocytes, either constitutively or upon induction by various stimuli $[7,25]$. The findings of this study corroborate the cytokine expression profiles of keratinocytes. The pro-inflammatory cytokines IL- 8 and TNF- $\alpha$ were expressed in the negative control cultures, suggesting that these cytokines are constitutively expressed. However, the expression of both cytokines was significantly higher in the positive control, indicating that higher levels of both IL- 8 and TNF- $\alpha$ could indicate skin irritation in vitro. High expression of TNF- $\alpha$ has been found in patients with keratinocyte lesions, rendering it important role in inflammatory process [26]. Elevated levels of TNF$\alpha$ expression could indicate activation of cytokine pathways associated with inflammation and disease progression [6]. CPSRTs did not induce increased production of either IL- 8 or TNF- $\alpha$, but maintained levels similar to those in the negative control, suggesting that these scaffolds did not provoke an irritation response in vitro.

Combining cells with natural or synthetic scaffolds is a fundamental approach in tissue engineering. The scaffolds help maintain a three-dimensional (3D) space for cells in order to facilitate cellular proliferation and differentiation and to eventually guide cellular organization into a defined architecture for tissue regeneration. Natural biopolymers have been the focus as they are biocompatible, biodegradable and come from renewable resources. Chitosan has structural characteristics similar to glycosaminoglycans, which can be useful for developing a skin replacement. Therefore, the CPSRT was produced in a skin-like format with a 3D porous structure to provide space for cellular ingrowth. Porous chitosan structures can be formed by freezing and lyophilizing chitosan salt solutions in suitable molds. During the freezing process, ice crystals nucleate along thermal gradients and generate a porous structure when removed by lyophilization.

Cultured pHDF cells adapted to the 3D environment by adhering to the $3 \mathrm{D}$ structure of the CPSRT at day 5 , as verified by live/dead staining and imaging by CLSM. 
This CPSRT supports cell attachment, as in agreement with Zhu et al. [27] and Fakhry et al. [28]. Cultured pHDFs grew rapidly and achieved confluence within the CPSRT at fourteen days post-seeding. This growth is likely due to the initial adaptation of cells to the $3 \mathrm{D}$ environment by adhering to the chitosan motifs in the construct. In addition, degraded chitosan oligomers have been effective at inducing cell migration and proliferation [29].

\section{Conclusion}

A naturally derived, bilayer porous biopolymer scaffold, termed a CPSRT, has been developed for skin tissue engineering with pore sizes ranging from 50 to $150 \mu \mathrm{m}$. It has been shown to be biocompatible, as it neither induced significant cytotoxic response in the $\mathrm{LDH}$ assay nor an irritation response in vitro. This biocompatibility is further supported by the observed ingrowth of pHDFs into the CPSRT.

\section{Acknowledgments}

This work was supported by a Grant (no. 03-03-01-0000PR0071/05) from the Intensification of Research in Priority Area Program (IRPA), Ministry of Science, Technology and Innovation (MOSTI) Malaysia, a Grant by International Atomic Energy Agency (IAEA) (no. 304/PPSP/6150097/ A127), and a Grant by Research University (RU) from Universiti Sains Malaysia (no. 1001/PPSP/812037).

\section{References}

[1] M. G. Peter, "Applications and environmental aspects of chitin and chitosan," Journal of Macromolecular Science: Pure and Applied Chemistry, vol. A32, pp. 629-640, 1995.

[2] M. N. V. Ravi Kumar, "A review of chitin and chitosan applications," Reactive and Functional Polymers, vol. 46, no. 1, pp. 1-27, 2000.

[3] M. Prabaharan, "Chitosan derivatives as promising materials for controlled drug delivery," Journal of Biomaterials Applications, vol. 23, no. 1, pp. 5-36, 2008.

[4] M. Morimoto, H. Saimoto, and Y. Shigemasa, "Control of functions of chitin and chitosan by chemical modification," Trends in Glycoscience and Glycotechnology, vol. 14, no. 78, pp. 205-222, 2002.

[5] C. K. Lim, A. S. Halim, H. Y. Lau, Z. Ujang, and A. Hazri, "In vitro cytocology model of oligo-chitosan and N, Ocarboxymethyl chitosan using primary normal human epidermal keratinocyte cultures," Journal of Applied Biomaterials \& Biomechanics, vol. 5, pp. 82-87, 2007.

[6] L. C. Keong and A. S. Halim, "In Vitro models in biocompatibility assessment for biomedical-grade chitosan derivatives in wound management," International Journal of Molecular Sciences, vol. 10, no. 3, pp. 1300-1313, 2009.

[7] C. K. Lim, N. S. Yaacob, Z. Ismail, and A. S. Halim, "In vitro biocompatibility of chitosan porous skin regenerating templates (PSRTs) using primary human skin keratinocytes," Toxicology in Vitro, vol. 24, no. 3, pp. 721-727, 2010.

[8] M. S. B. Abdull Rasad, A. S. Halim, K. Hashim, A. H. A. Rashid, N. Yusof, and S. Shamsuddin, "In vitro evaluation of novel chitosan derivatives sheet and paste cytocompatibility on human dermal fibroblasts," Carbohydrate Polymers, vol. 79, no. 4, pp. 1094-1100, 2010.
[9] D. K. Singh and A. R. Ray, "Biomedical applications of chitin, chitosan, and their derivatives," Reviews in Macromolecular Chemistry and Physics, vol. 40, pp. 69-83, 2000.

[10] I. Zainol, S. M. Ghani, A. Mastor, M. A. Derman, and M. F. Yahya, "Enzymatic degradation study of porous chitosan membrane," Materials Research Innovations, vol. 13, no. 3, pp. 316-319, 2009.

[11] M. Coufal, M. M. Maxwell, D. E. Russel et al., "Discovery of a novel small-molecule targeting selective clearance of mutant huntingtin fragments," Journal of Biomolecular Screening, vol. 12, no. 3, pp. 351-360, 2007.

[12] H. Liu, H. Fan, Y. Cui, Y. Chen, K. Yao, and J. C. H. Goh, "Effects of the controlled-released basic fibroblast growth factor from chitosan-gelatin microspheres on human fibroblasts cultured on a chitosan-gelatin scaffold," Biomacromolecules, vol. 8, no. 5, pp. 1446-1455, 2007.

[13] H. Liu, Y. Yin, and K. Yao, "Construction of chitosan-gelatinhyaluronic acid artificial skin in vitro," Journal of Biomaterials Applications, vol. 21, no. 4, pp. 413-430, 2007.

[14] H. R. Lin, K. S. Chen, S. C. Chen et al., "Attachment of stem cells on porous chitosan scaffold crosslinked by Na5P3O10," Materials Science and Engineering C, vol. 27, no. 2, pp. 280284, 2007.

[15] X. Ponsoda, C. Núñez, J. V. Castell, and M. J. Gómez-Lechón, "Evaluation of the cytotoxic effects of MEIC chemicals 31-50 on primary culture of rat hepatocytes and hepatic and nonhepatic cell lines," ATLA Alternatives to Laboratory Animals, vol. 25, no. 4, pp. 423-436, 1997.

[16] A. Yang, D. L. Cardona, and F. A. Barile, "Subacute cytotoxicity testing with cultured human lung cells," Toxicology in Vitro, vol. 16, no. 1, pp. 33-39, 2002.

[17] C. Clemedson, F. A. Barile, C. Chesné et al., "MEIC evaluation of acute systemic toxicity. Part VII. Prediction of human toxicity by results from testing of the first 30 reference chemicals with 27 further in vitro assays," ATLA Alternatives to Laboratory Animals, vol. 28, supplement 1, pp. 161-200, 2000.

[18] G. R. Sharpe and C. Fisher, "Time-dependent inhibition of growth of human keratinocytes and fibroblasts by cyclosporin A: effect on keratinocytes at therapeutic blood levels," British Journal of Dermatology, vol. 123, no. 2, pp. 207-213, 1990.

[19] C. Baba, K. Yanagida, T. Kanzaki, and M. Baba, "Colorimetric lactate dehydrogenase (LDH) assay for evaluation of antiviral activity against bovine viral diarrhoea virus (BVDV) in vitro," Antiviral Chemistry and Chemotherapy, vol. 16, no. 1, pp. 3339, 2005.

[20] Y. W. Cho, Y. N. Cho, S. H. Chung, G. Yoo, and S. W. Ko, "Water-soluble chitin as a wound healing accelerator," Biomaterials, vol. 20, no. 22, pp. 2139-2145, 1999.

[21] D. W. Lee, S. A. Shirley, R. F. Lockey, and S. S. Mohapatra, "Thiolated chitosan nanoparticles enhance anti-inflammatory effects of intranasally delivered theophylline," Respiratory Research, vol. 7, article no. 112, 2006.

[22] I. Setnikar, R. Cereda, M. A. Pacini, and L. Revel, "Antireactive properties of glucosamine sulfate," Arzneimittel-Forschung, vol. 41, no. 2, pp. 157-161, 1991.

[23] T. Mammone, D. Gan, C. Fthenakis, and K. Marenus, "The effect of N-acetyl-glucosamine on stratum corneum desquamation and water content in human skin," Journal of Cosmetic Science, vol. 60, no. 4, pp. 423-428, 2009.

[24] D. N. Sauder, "The role of epidermal cytokines in inflammatory skin diseases," Journal of Investigative Dermatology, vol. 95, no. 5, pp. 27-28, 1990.

[25] A. Oxholm, M. Diamant, P. Oxholm, and K. Bendtzen, "Interleukin-6 and tumour necrosis factor alpha are expressed 
by keratinocytes but not by Langerhans cells," APMIS, vol. 99, no. 1, pp. 58-64, 1991.

[26] D. Daliani, R. A. Ulmer, C. Jackow et al., "Tumor necrosis factor- $\alpha$ and interferon- $\gamma$, but not HTLV-I tax, are likely factors in the epidermotropism of cutaneous T-cell lymphoma via induction of interferon-inducible protein-10," Leukemia and Lymphoma, vol. 29, no. 3-4, pp. 315-328, 1998.

[27] A. P. Zhu, S. Q. Wang, D. M. Cheng et al., "Attachment and growth of cultured fibroblast cells on chitosan/PHEA-blended hydrogels," Chinese Journal of Biotechnology, vol. 18, no. 1, pp. 109-111, 2002.

[28] A. Fakhry, G. B. Schneider, R. Zaharias, and S. Şenel, "Chitosan supports the initial attachment and spreading of osteoblasts preferentially over fibroblasts," Biomaterials, vol. 25, no. 11, pp. 2075-2079, 2004.

[29] Y. Okamoto, A. Inoue, K. Miyatake, K. Ogihara, Y. Shigemasa, and S. Minami, "Effects of chitin/chitosan and their oligomers/ monomers on migrations of macrophages," Macromolecular Bioscience, vol. 3, no. 10, pp. 587-590, 2003. 

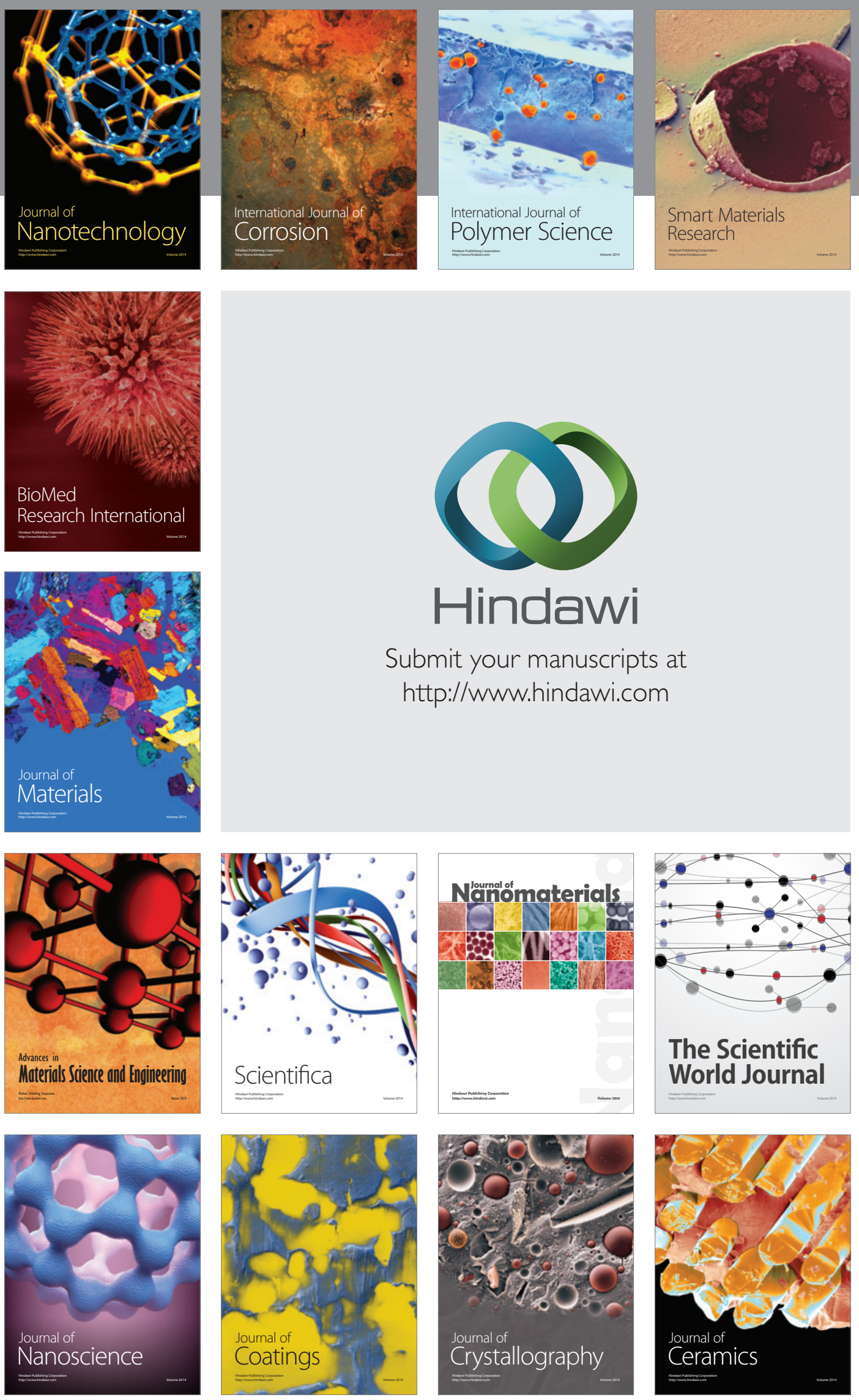

The Scientific World Journal

Submit your manuscripts at

http://www.hindawi.com

\section{World Journal}

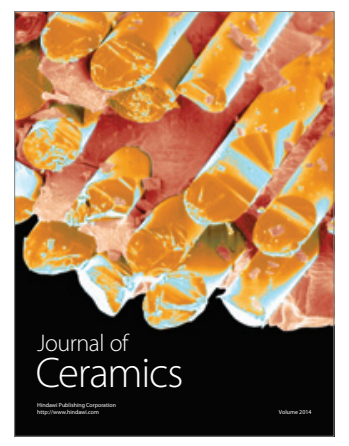

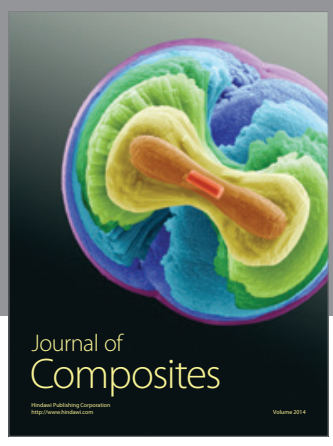
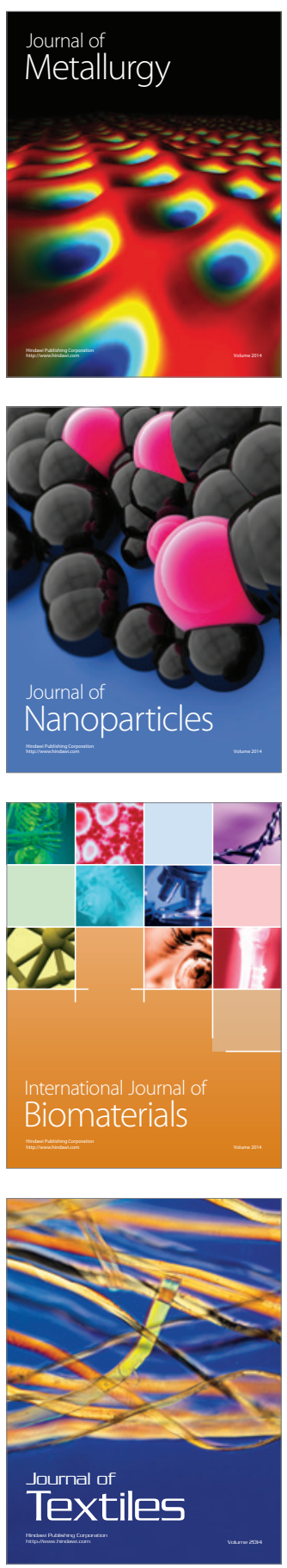